\title{
Effects of Monoamine Oxidase Inhibition on the Reinforcing Properties of Low-Dose Nicotine
}

\author{
Tracy T Smith*,', Laura E Rupprecht ${ }^{2}$, Samantha N Cwalina', Matthew J Onimus ${ }^{3}$, Sharon E Murphy ${ }^{4}$, \\ Eric C Donny' and Alan F Sved ${ }^{1,3}$ \\ 'Department of Psychology, University of Pittsburgh, Pittsburgh, PA, USA; ${ }^{2}$ Center for Neuroscience, University of Pittsburgh, Pittsburgh, PA, USA; \\ ${ }^{3}$ Department of Neuroscience, University of Pittsburgh, Pittsburgh, PA, USA; ${ }^{4}$ Department of Biological Sciences, University of Minnesota, \\ Minneapolis, MN, USA
}

\begin{abstract}
The Food and Drug Administration (FDA) has the authority to regulate cigarette smoke constituents, and a reduction in nicotine content might benefit public health by reducing the prevalence of smoking. Research suggests that cigarette smoke constituents that inhibit monoamine oxidase (MAO) may increase the reinforcing value of low doses of nicotine. The aim of the present experiments was to further characterize the impact of MAO inhibition on the primary reinforcing and reinforcement enhancing effects of nicotine in rats. In a series of experiments, rats responded for intravenous nicotine infusions or a moderately-reinforcing visual stimulus in daily I-h sessions. Rats received pre-session injections of known MAO inhibitors. The results show that ( 1 ) tranylcypromine (TCP), a known MAO inhibitor, increases sensitivity to the primary reinforcing effects of nicotine, shifting the dose-response curve for nicotine to the left, (2) inhibition of MAO-A, but not MAO-B, increases low-dose nicotine self-administration, (3) partial MAO-A inhibition, to the degree observed in chronic cigarette smokers, also increases low-dose nicotine self-administration, and (4) TCP decreases the threshold nicotine dose required for reinforcement enhancement. The results of the present experiments suggest cigarette smoke constituents that inhibit MAO-A, in the range seen in chronic smokers, are likely to increase the primary reinforcing and reinforcement enhancing effects of low doses of nicotine. If the FDA reduces the nicotine content of cigarettes, then variability in constituents that inhibit MAO-A could impact smoking.

Neuropsychopharmacology (2016) 4I, 2335-2343; doi:I0.1038/npp.2016.36; published online 6 April 2016
\end{abstract}

\section{INTRODUCTION}

In 2009, the Food and Drug Administration (FDA) was given the authority to regulate the content of tobacco constituents (U.S. Congress, 2009). Reducing the nicotine content in cigarettes to a non-addictive level has been suggested as a potential strategy for improving public health because it may decrease the prevalence of smoking (Benowitz and Henningfield, 1994; Hatsukami et al, 2010). A recently completed clinical trial on nicotine reduction showed that individuals who smoked very low nicotine content cigarettes for 6 weeks smoked fewer cigarettes and scored lower on measures of nicotine dependence compared with individuals who smoked cigarettes with a normal nicotine content

\footnotetext{
*Correspondence: Dr TT Smith, Department of Psychology, University of Pittsburgh, 4I 20 Sennott Square, 210 S. Bouquet Street, Pittsburgh, PA 15260, USA, Tel: +I 412 624 8525, Fax: + I 4126244428 , E-mail: tracysmith@pitt.edu

Portions of these data were presented as part of presentations at the annual meeting for the Society for Nicotine and Tobacco Research in Seattle, WA on 6 February 2014, the Society for Nicotine and Tobacco Research in Philadelphia, PA on 25-28 February 20I5, and the annual meeting for the Association for Behavior Analysis in Chicago, IL on 26 May 2014.

Received 27 November 20 15; revised I5 February 2016; accepted 29 February 2016; accepted article preview online 9 March 2016
}

(Donny et al, 2015). However, the impact of non-nicotine cigarette constituents on a potential nicotine reduction policy is unknown, and clinical researchers lack the tools necessary to investigate whether non-nicotine constituents might moderate the impact of nicotine reduction.

There are over 8000 non-nicotine constituents in cigarette smoke (Rodgman and Perfetti, 2013), and researchers have used a rodent model of nicotine self-administration to investigate whether these constituents might reinforce behavior or interact with nicotine to reinforce behavior (Arnold et al, 2014; Bardo et al, 1999; Belluzzi et al, 2005; Brennan et al, 2015; Clemens et al, 2009; Costello et al, 2014; Smith et al, 2015). Cigarette smoke constituents have been shown to inhibit monoamine oxidase (MAO), an enzyme involved in the oxidative degradation of monoamines. Both MAO isozymes, MAO-A and MAO-B, are inhibited by an average of $28-40 \%$ in chronic smokers (Fowler et al, 1996a, b). The impact of this inhibition on behavior following a reduction in nicotine content is unknown. Tobacco types and cigarette brands have also been shown to vary in the degree to which they inhibit MAO (Lewis et al, 2012), so it is important to understand the impact of MAO inhibition on the reinforcing value of nicotine, especially on low nicotine doses.

Previous research has investigated the impact of MAO inhibition on the reinforcing effects of nicotine in rats by delivering drugs known to inhibit MAO, such as 
tranylcypromine (TCP). MAO inhibition has been shown to increase nicotine self-administration on fixed-ratio (FR) and progressive-ratio schedules (Guillem et al, 2005; Smith et al, 2015; Villegier et al, 2007). However, the high doses of TCP used by some researchers have been shown to increase nicotine self-administration through acute, off-target effects (eg, monoamine release) rather than the long-lasting effect of MAO inhibition (Lotfipour et al, 2011; Villegier et al, 2011). We have recently shown that at more moderate doses $(1 \mathrm{mg} /$ $\mathrm{kg}$ ), the impact of TCP on nicotine self-administration is likely dependent on MAO inhibition and not these off-target effects (Smith et al, 2015).

Although MAO inhibition clearly increases the primary reinforcing effect of nicotine under some conditions (Guillem et al, 2005; Smith et al, 2015), more research is needed. First, the impact of MAO inhibition across different nicotine doses is not fully understood. Recent research from our laboratory has shown that the impact of $\mathrm{MAO}$ inhibition on nicotine self-administration appears to be selective to low nicotine doses (Smith et al, 2015), and previous research has suggested that the effect of MAO inhibition on nicotine self-administration may actually reverse at high nicotine doses (Guillem et al, 2005). Second, research using selective MAO-A and MAO-B inhibitors has suggested that it is MAO-A, and not MAO-B, inhibition that increases nicotine self-administration (Guillem et al, 2006), although MAO activity was not measured to confirm selective inhibition of MAO isoforms. Third, the majority of research in this area has used large doses of MAO inhibitors, which likely fully inhibit MAO (Guillem et al, 2005, 2006; Villegier et al, 2003, 2006, 2007, 2011). Thus, it is unknown whether partial inhibition, in the range seen in chronic smokers, increases the reinforcing value of nicotine. Finally, all previous self-administration research in this area has focused on the impact of MAO inhibition on the primary reinforcing effect of nicotine. However, we have previously shown that rats will respond for a visual stimulus (VS) consisting of a 1-s cue light presentation and a 1-min white houselight offset, and that responding for the VS is increased in rats receiving non-contingent infusions of nicotine (Caggiula et al, 2009; Donny et al, 2003; Palmatier et al, 2006; Rupprecht et al, 2015). The action of nicotine to increase the value of other reinforcers is known as reinforcement enhancement. The reinforcement enhancement effect has been shown to be as important or more important than the primary reinforcing effect of nicotine for nicotine self-administration (Caggiula et al, 2009). The impact of MAO inhibition on the reinforcement enhancing effect of nicotine is unknown.

The goal of the present studies was to extend our knowledge about the impact of MAO inhibition on the primary reinforcing and reinforcement enhancing effects of nicotine. We focused on low nicotine doses because of the relevance of low-dose nicotine reinforcement to current research priorities of the FDA and the large impact that has been previously reported of TCP on low doses (Smith et al, 2015). Our studies aimed to (1) characterize the impact of MAO inhibition on nicotine reinforcement across the nicotine dose-response curve, (2) investigate whether inhibition of MAO-A or MAO-B is responsible for the increase in low-dose nicotine self-administration by using selective MAO-A and MAO-B inhibitors, confirming selectivity following the experiment, (3) assess whether partial MAO inhibition increases low-dose nicotine selfadministration, and (4) investigate the impact of MAO inhibition on the threshold nicotine dose for reinforcement enhancement.

\section{MATERIALS AND METHODS}

\section{Subjects}

Male Sprague-Dawley rats (Harlan-Farms, IN) weighing between 200 and $225 \mathrm{~g}$ upon arrival were used as subjects. Rats were individually housed in wire-mesh, hanging cages, or tub cages on a ventilated rack in a temperature-controlled colony room. Rats were kept on a reverse light-dark $12: 12$-h schedule, and testing occurred during the dark phase. Rats received ad libitum chow for the first 7 days while habituating to individual home cages and access to ad libitum water in the home cages throughout the experiments. At least 8 days after arrival, rats were implanted with jugular catheters and were changed to a feeding schedule where $20 \mathrm{~g} /$ day was delivered after each daily self-administration session. Rats were allowed at least 5 days of recovery following surgery.

\section{Apparatus}

Standard self-administration operant chambers (ENV-008 CT; Med-Associates) were configured as previously described (Smith et al, 2013), and included two nosepoke holes below two stimulus lights on one side of the chamber. For self-administration experiments, the houselight was lit red during the session, and in the experiment on reinforcement enhancement the houselight was lit white during the session and turned off as part of the VS reinforcer (details below).

\section{Drugs}

Nicotine hydrogen tartrate salt (Sigma, St Louis, MO) was dissolved in $0.9 \%$ saline (doses expressed as free base), and delivered intravenously (Experiments 1-3) or subcutaneously (s.c., Experiment 4). Tranylcypromine hydrochloride (TCP, Sigma), pargyline hydrochloride (pargyline, Sigma), $N$-Methyl- $N$-propargyl-3-(2,4-dichlorophenoxyl) propylamine hydrochloride (clorgyline, Sigma), and rasagiline mesylate (rasagiline, Tocris Bioscientific, Minneapolis, MN) were dissolved in $0.9 \%$ saline and delivered intraperitoneally (i.p.) 1-h before each self-administration session in a volume of $1 \mathrm{ml} / \mathrm{kg}$. All solutions were sterilized by being passed through a $0.22-\mu \mathrm{m}$ filter. The $\mathrm{pH}$ of solutions delivered i.p. or s.c. was adjusted to $7.0 \pm 0.2$.

\section{Assay of MAO Activity}

MAO activity was measured in vitro in supernatant fluid from homogenates of the left dorsal striatum using an absorbance-based assay involving the oxidation of p-tyramine coupled to the conversion of Ampliflu Red (Sigma) to resorufin in the presence of horseradish peroxidase as previously described (Smith et al, 2015). MAO-B activity was determined in the presence of clorgyline in the assay, and MAO-A was calculated as the difference between MAO$\mathrm{B}$ activity and total activity. 


\section{Assay of Nicotine Levels in Brain and Blood}

For the quantitation of the nicotine, $100 \mu$ plasma or brain homogenate (cortical tissue punches weighing $\sim 5 \mathrm{mg}$ homogenized in $500 \mu \mathrm{l}$ of water) was mixed with $450 \mu \mathrm{l}$ of $1.5 \%$ formic acid in water and $\mathrm{D}_{3}$-internal standards, loaded on a 96-well Oasis MCX solid phase extraction plate (2 mg; Waters Corporation, Milford, MA) that had been conditioned with methanol and $1.5 \%$ formic acid. The plates were washed with $0.5 \mathrm{ml}$ of $1.5 \%$ formic acid in water and methanol, then nicotine and metabolites were eluted with $50 \mu$ of $5 \%$ ammonium hydroxide in methanol. Samples were evaporated to dryness under a gentle stream of nitrogen, re-suspended in $25 \mu \mathrm{l}$ of $100 \mathrm{mM}$ ammonium acetate in methanol and assayed by LC tandem mass spectrometry (LC/MS/MS) as previously described (Murphy et al, 2014).

\section{Procedures for Self-Administration Experiments}

Surgery. Procedures for jugular catheterization were as previously described (Smith et al, 2013). For the first 5 days following surgery, rats had their catheter flushed once-daily with $0.1 \mathrm{ml}$ of a sterile saline solution containing heparin ( $3 \mathrm{U})$, an antibiotic, and streptokinase (833.3 U) to maintain catheter patency and prevent infection. The antibiotic changed depending on drug availability and was either timentin $(6.67 \mathrm{mg})$ or cefazolin $(10 \mathrm{mg})$. After this initial post-surgery time period, the flushing solution contained only the heparin and the antibiotic. Catheter patency was tested at the end of each experiment $(5 \mathrm{mg} / \mathrm{kg}$ intravenous methohexital), and data are only shown from rats that passed the patency test.

Procedures. Rats were given the opportunity to respond via nosepokes for i.v. infusions. The side of the active nosepoke hole (left $v s$ right) was counterbalanced across rats. Active pokes resulted in a simultaneous onset of an intravenous infusion, a 15-s cue light presentation and 1-min time-out period according to the reinforcement schedule in effect. Active nosepokes during time out and inactive nosepokes were recorded, but had no consequence. Sessions lasted $1 \mathrm{~h}$ and were conducted 7 days per week.

\section{Experiment 1: Impact of MAO Inhibition on the Dose-Response Curve for Nicotine Self-Administration}

The aim of this experiment was to characterize the impact of MAO inhibition on nicotine self-administration across a wide range of nicotine doses. Rats received a pre-session i.p. injection of either TCP $(1.0 \mathrm{mg} / \mathrm{kg})$ or saline $1 \mathrm{~h}$ before the start of the self-administration session. Across 56 sessions, rats were given the opportunity to respond on an FR2 schedule of reinforcement for intravenous nicotine. The nicotine dose was increased every seven sessions: $0,1.875,3.75,7.5,15,30,60$, and $90 \mu \mathrm{g} / \mathrm{kg} /$ infusion. Following completion of all doses, rats were tested on a progressive ratio schedule in which the number of responses required to earn an infusion increased with each infusion $(1,3$, $6,10,15,20,25,32,40,50,62,77,95,118,145,179,219,268$, 328, 402, and 492) (Depootere et al, 1993). Rats completed four sessions in which the nicotine dose was $90 \mu \mathrm{g} / \mathrm{kg} /$ infusion, and four sessions in which the nicotine dose was reduced to $15 \mu \mathrm{g} /$ $\mathrm{kg} /$ infusion. The first three of the four sessions at each dose ended after $2 \mathrm{~h}$, and the last session ended after $4 \mathrm{~h}$. Data shown are the average of the last two 2-h sessions, but the data from the 4 -h session were consistent with these data. A follow-up experiment tested the impact of a different MAO inhibitor (pargyline) on a low dose of nicotine $(10 \mu \mathrm{g} / \mathrm{kg} /$ infusion $)$ over 20 sessions on an FR2 schedule of reinforcement.

\section{Experiment 2: Impact of Selective MAO Inhibitors on Low-Dose Nicotine Self-Administration}

Our treatment regimen of TCP has been shown to nearly completely inhibit both MAO-A and MAO-B (Smith et al, 2015). The aim of this experiment was to use selective MAO$\mathrm{A}$ and MAO-B inhibitors to investigate the role of inhibition of each isozyme on low-dose nicotine self-administration. Rats were given the opportunity to respond on an FR2 schedule of reinforcement for $10 \mu \mathrm{g} / \mathrm{kg} /$ infusion nicotine during 20 daily sessions. Rats received an i.p. injection 1-h before the session of either $1.0 \mathrm{mg} / \mathrm{kg}$ clorgyline (a selective MAO-A inhibitor), $0.05 \mathrm{mg} / \mathrm{kg}$ rasagiline (a selective MAO-B inhibitor), a solution containing both $1.0 \mathrm{mg} / \mathrm{kg}$ clorgyline and $0.05 \mathrm{mg} / \mathrm{kg}$ rasagline, or saline.

One hour after the final i.p. injection, rats were anesthetized with isoflurane and decapitated. The left dorsal striatum was dissected and flash frozen before being assayed for MAO activity. Tissue collection occurred 3 days after the 20th self-administration session though rats continued to receive their assigned injection each day.

\section{Experiment 3: Impact of Partial MAO Inhibition on Low-Dose Nicotine Self-Administration}

The dose of TCP used in the nicotine dose-response experiment produces near complete MAO inhibition (Smith et al, 2015). However, MAO activity is only partially inhibited, on average $28-40 \%$, in chronic smokers (Fowler et al, 1996a, b). Thus, Experiment 3 assessed the impact of partial MAO inhibition on low-dose nicotine self-administration $(10 \mu \mathrm{g} /$ $\mathrm{kg} /$ infusion). Rats received $1.0 \mathrm{mg} / \mathrm{kg}$ TCP, $0.3 \mathrm{mg} / \mathrm{kg}$ TCP, $0.1 \mathrm{mg} / \mathrm{kg}$ TCP, or saline $1 \mathrm{~h}$ before the session. These doses were expected to fully or partially inhibit MAO. Rats were given 20 sessions to respond on an FR2 schedule of reinforcement, after which the FR schedule was changed to an FR5 (10 sessions), followed by an FR1 (7 sessions).

At the end of the behavioral experiment, these rats were utilized for two purposes: (1) testing the impact of these TCP doses on MAO activity and (2) testing whether TCP alters nicotine blood or brain levels. On the day following the last day of self-administration, rats received their assigned presession injection and completed a 1 -h session during which they received infusions of a low nicotine dose $(10 \mu \mathrm{g} / \mathrm{kg} /$ infusion) to model self-administration. To ensure all rats received equivalent exposure to nicotine, infusions were not contingent upon responding. The number of infusions was matched to the average number of infusions earned in all groups over the last 3 days of self-administration in Experiment $3(10 \mu \mathrm{g} / \mathrm{kg} /$ infusion, 22 infusions), and infusions were delivered at regularly spaced intervals (every $162 \mathrm{~s}$ ). Rats were killed within 5 min of the end of the session (approximately $2 \mathrm{~h}$ after TCP injection). The left dorsal striatum was dissected to assay MAO activity, and trunk blood and remaining brain were collected to assay nicotine 
levels. A random subset of rats in the $1.0-\mathrm{mg} / \mathrm{kg}$ TCP and saline groups was used for the nicotine assay.

\section{Experiment 4: Impact of MAO Inhibition on Threshold for Reinforcement Enhancement}

To examine the reinforcement enhancement action of nicotine, we utilized an approach in which rats responded for a mildly reinforcing VS (1-min offset of a white houselight, 1-s cue light onset) while receiving noncontingent injections of nicotine (Donny et al, 2003). In a $2 \times 2$ design, all rats received an i.p. injection $1 \mathrm{~h}$ before the session of either TCP $(1 \mathrm{mg} / \mathrm{kg})$ or saline, and an s.c. injection $5 \mathrm{~min}$ before the session of either nicotine or saline. This created four groups: TCP/NIC, TCP/SAL, SAL/NIC, and SAL/SAL. The nicotine dose was increased across sessions: $0.03 \mathrm{mg} / \mathrm{kg}$ (10 sessions), $0.1 \mathrm{mg} / \mathrm{kg}$ (5 sessions), and $0.3 \mathrm{mg} / \mathrm{kg}$ (4 sessions). The first two doses $(0.03,0.1 \mathrm{mg} / \mathrm{kg})$ are relatively low s.c. doses of nicotine not expected to enhance the value of the VS on their own (unpublished observations), and the highest nicotine dose $(0.3 \mathrm{mg} / \mathrm{kg})$ is a dose in the range expected to enhance the value of the VS (Buffalari et al, 2014; Weaver et al, 2012; Wing and Shoaib, 2010). For this experiment, sessions were conducted 5 days per week.

\section{Data Analysis}

The primary outcome in all behavioral experiments was the average number of infusions or VS presentations earned over the last 2 (PR schedule) or 3 (FR schedules) days of self-administration. Omnibus ANOVA tests were followed by planned comparisons using paired-samples or independent-samples $t$-tests when appropriate $(\alpha=0.05)$. Statistical analyses were conducted on the MAO activity measure, but graphs show MAO activity in TCP groups expressed as a percent of average activity in the saline group.

\section{RESULTS}

\section{Experiment 1: Impact of MAO Inhibition on the Dose-Response Curve for Nicotine Self-Administration}

TCP shifts the dose-response curve for nicotine to the left (Figure 1a). A $2 \times 8$ ANOVA confirmed there was a main effect of nicotine dose $(F(7,161)=19.87, p<0.001)$, and a significant nicotine dose $\times$ pre-session injection interaction $(F(7,161)=4.35, p=0.014)$. Independent-samples $t$-tests confirmed that rats receiving TCP injections earned significantly more infusions at relatively low nicotine doses $(7.5 \mu \mathrm{g} /$ $\mathrm{kg} /$ infusion: $t \quad(26)=2.217, \quad p=0.038 ; 15 \mu \mathrm{g} / \mathrm{kg} /$ infusion: a
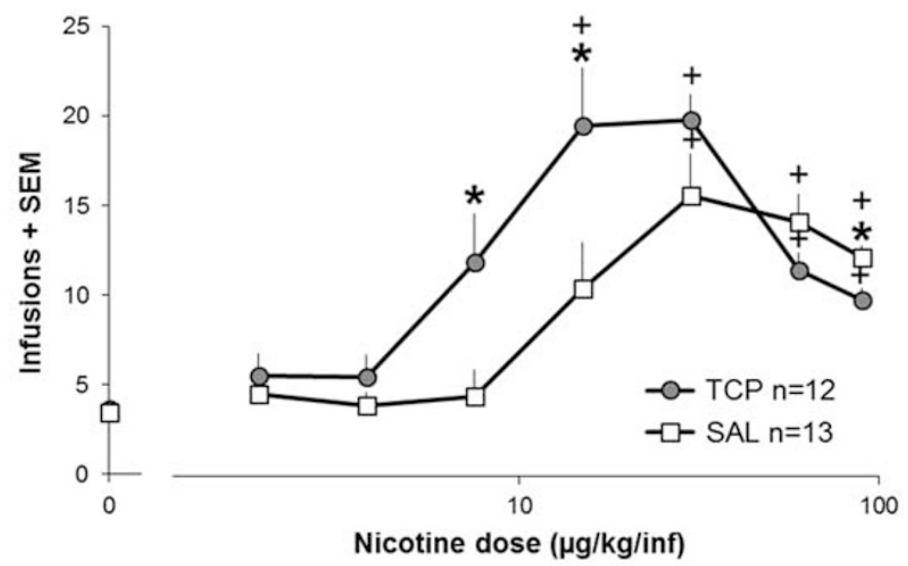

b
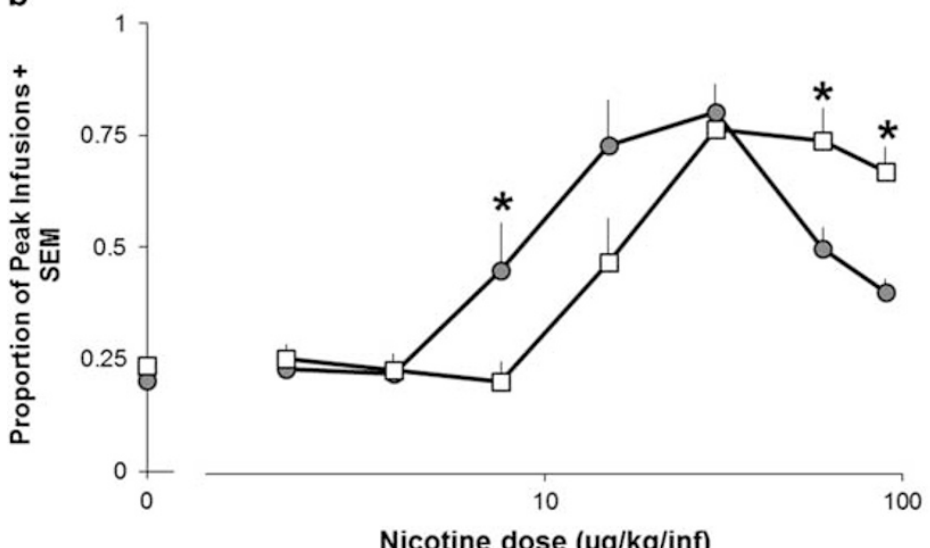
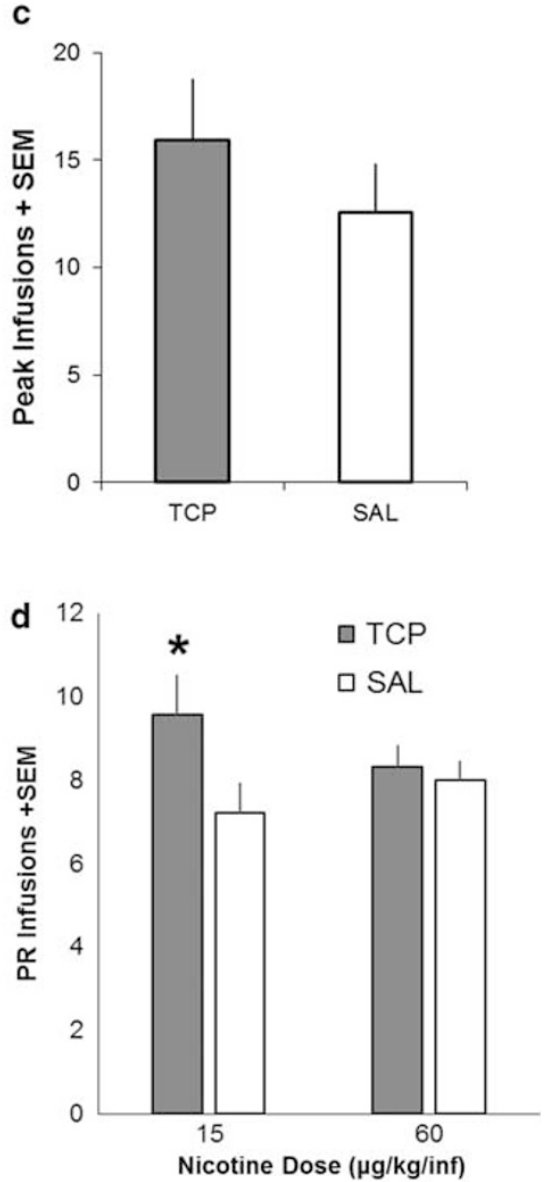

Figure I Average infusions over the last 3 days of self-administration at each nicotine dose (a), peak number of infusions earned across all doses (b), average infusions expressed as a proportion of each rat's peak number of earned infusions (c) and average infusions earned on the last 2 days on a PR schedule (d). A significant difference between the TCP group and the saline group at a single nicotine dose is represented by *, and a significant difference from $0.0 \mu \mathrm{g} / \mathrm{kg} /$ infusion is represented by + . 
$t(26)=2.10, p=0.046)$ and significantly fewer infusions at a relatively high nicotine dose $(90 \mu \mathrm{g} / \mathrm{kg} /$ infusion: $t(23)=$ 2.425, $p=0.024)$. TCP also shifted the threshold dose of nicotine required for self-administration. Paired-samples $t$-test confirmed that rats receiving TCP earned more nicotine infusions at nicotine doses $7.5 \mu \mathrm{g} / \mathrm{kg} /$ infusion or greater than they earned at $0.0 \mu \mathrm{g} / \mathrm{kg} /$ infusion nicotine $(p s<0.05)$, while rats receiving saline injections earned more infusions when the dose was at least $30 \mu \mathrm{g} / \mathrm{kg} /$ infusion than they did at $0.0 \mu \mathrm{g} / \mathrm{kg} /$ infusion nicotine $(p s<0.05)$. The pattern for active responding was consistent with the pattern for infusions, and there were no significant differences between the groups for inactive responding (data not shown).

The difference in earned infusions between rats receiving TCP and saline at higher nicotine doses appears relatively small. However, there is a large decrease in earned infusions at these doses for rats receiving TCP compared with the peak of the dose-response curve, while these doses are near the peak of the curve for rats receiving saline. To illustrate the change from the peak of the dose-response curve, Figure $1 \mathrm{~b}$ expresses infusions earned at each dose as a proportion of the peak number of infusions earned for each rat (average of last 3 days at each dose). The peak number of infusions trended toward being significantly greater for rats receiving TCP $(t(23)=2.00, p=0.057$, Figure 1c). Independent-samples $t$-tests confirmed the same pattern shown in Figure 1a ( $p s<0.05)$, suggesting that the proportional decrease at high doses is larger for rats receiving TCP.

Independent-samples $t$-tests revealed that TCP increased the number of infusions earned on a PR schedule when the nicotine dose is low $(15 \mu \mathrm{g} / \mathrm{kg} /$ infusion, $t \quad(23)=2.27$, $p<0.05)$, but had no effect when the nicotine dose was high (90 $\mathrm{gg} / \mathrm{kg} /$ infusion) (Figure 1d). The pattern for active responding was consistent with the pattern for infusions, but there was no significant difference between the groups for inactive responding (data not shown).

In a follow-up experiment, pargyline significantly increased the number of infusions of $10 \mu \mathrm{g} / \mathrm{kg} /$ infusion earned (pargyline mean infusions $=21.4, n=8$; saline mean infusions $=6.3, n=8$ ) and inhibited both MAO-A and MAO-B activity by greater than $85 \%$ (data not shown). There was no significant impact on inactive responding (pargyline mean inactive responses $=12.5$, saline mean inactive responses $=4.7$ ).

\section{Experiment 2: Impact of Selective MAO Inhibitors on Low-Dose Nicotine Self-Administration}

The selective MAO-A inhibitor clorygline increased selfadministration of $10 \mu \mathrm{g} / \mathrm{kg} /$ infusion, while the selective MAO-B inhibitor rasagiline did not (Figure 2a). A $2 \times 2$ (clorgyline $\times$ rasagiline) ANOVA revealed a main effect of clorgyline $(F(1,32)=7.97, p=0.008)$, but no main effect of rasagiline $(p>0.05)$, and no interaction $(p>0.05)$. The pattern was the same for active responding, but there were no significant effects for inactive responding. The impact of clorgyline on self-administration appears after 5-10 sessions of self-administration. MAO-A activity was significantly decreased in both groups receiving clorgyline injections $(p s<0.05)$, and MAO-B activity was significantly decreased in all groups receiving rasagiline injections $(p s<0.05)$ (Figure 2b). Rats receiving rasagiline injections also had significantly increased MAO-A activity compared with rats receiving saline injections $(p<0.05)$.

\section{Experiment 3: Impact of Partial MAO Inhibition on Low-Dose Nicotine Self-Administration}

A $3 \times 4$ ANOVA revealed a significant main effect of FR $(F(2,128)=107.13, p<0.001)$ and group $(F(3,64)=3.41$, $p=0.023)$, but no significant interaction $(F(6128)=0.95$, $p>0.05$ ) (Figure 3b). Follow-up independent-samples $t$-tests confirmed that rats receiving $1.0 \mathrm{mg} / \mathrm{kg}$ TCP earned more nicotine infusions on an FR2 $(t(33)=2.44, p=0.021)$ and FR5 $(t(33)=2.55, p=0.016)$, and rats receiving $0.3 \mathrm{mg} / \mathrm{kg}$ TCP earned significantly more infusions at all FRs (FR2: $t(32)=3.87, p=0.001$; FR5: $t(32)=2.26, p=0.031$; FR1: $t(32)=2.42, p=0.032)$. There was a trend for $0.1 \mathrm{mg} / \mathrm{kg}$ TCP to increase nicotine self-administration on an FR2 $(t(31)=1.796, p=0.082)$. The pattern was the similar for active responding, but there were no significant effects for inactive responding. Figure $3 \mathrm{c}$ shows the impact of $1.0 \mathrm{mg} /$ $\mathrm{kg}, 0.3 \mathrm{mg} / \mathrm{kg}$, and $0.1 \mathrm{mg} / \mathrm{kg}$ TCP on MAO activity. MAO-B and total MAO activity were significantly inhibited in all groups. MAO-A was significantly inhibited in the $1.0-$ and
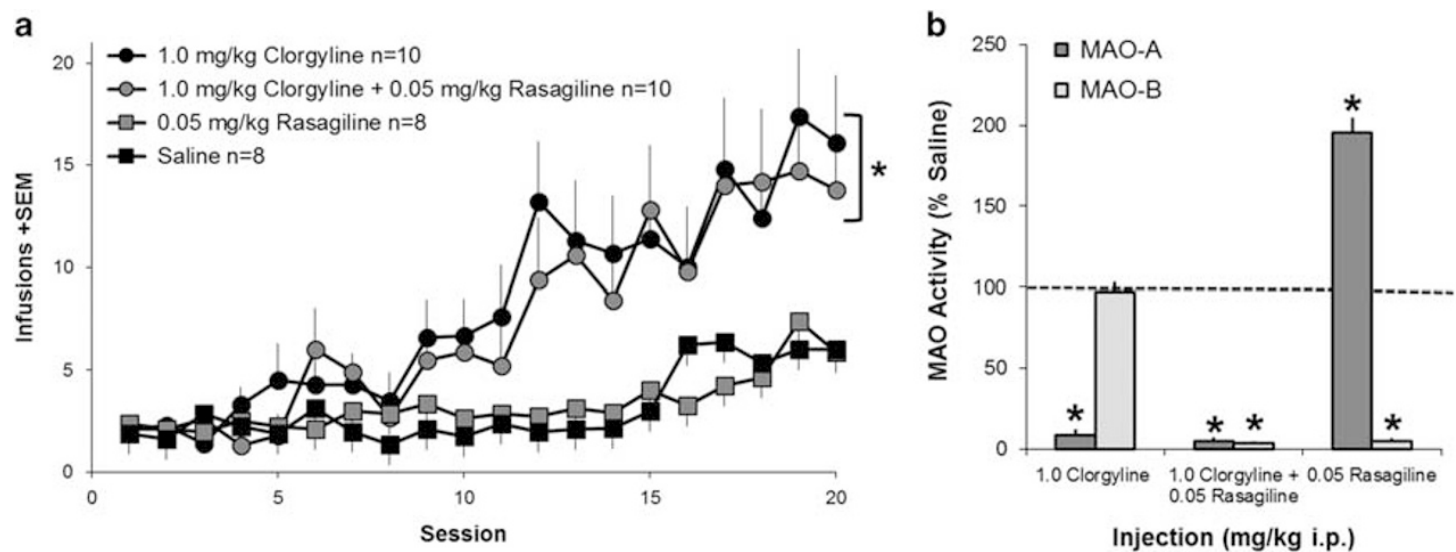

Figure 2 Average infusions ( \pm SEM) earned across 20 self-administration sessions (a). A significant main effect of clorgyline is represented by *. Average MAO-A and MAO-B activity expressed as a percent of activity in saline control rats (b). A significant difference in activity from saline represented by *. 
a

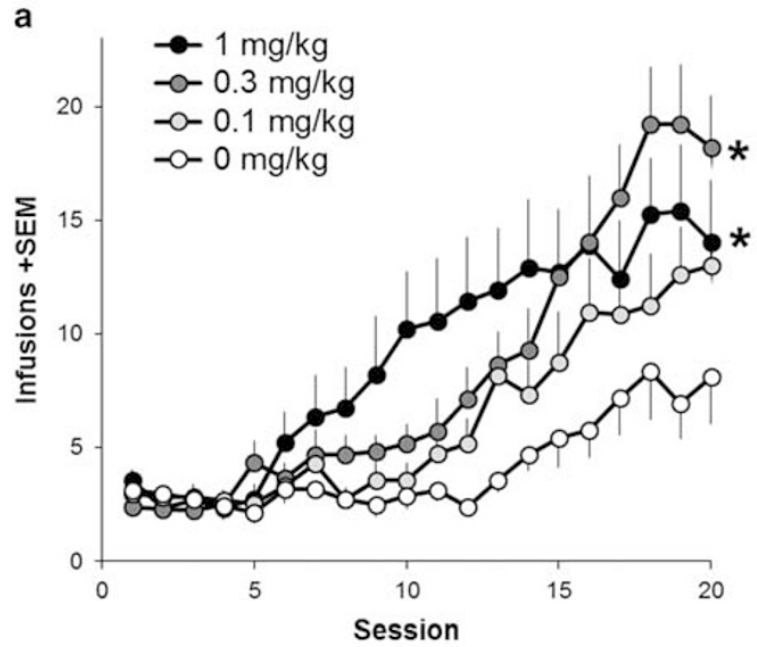

C

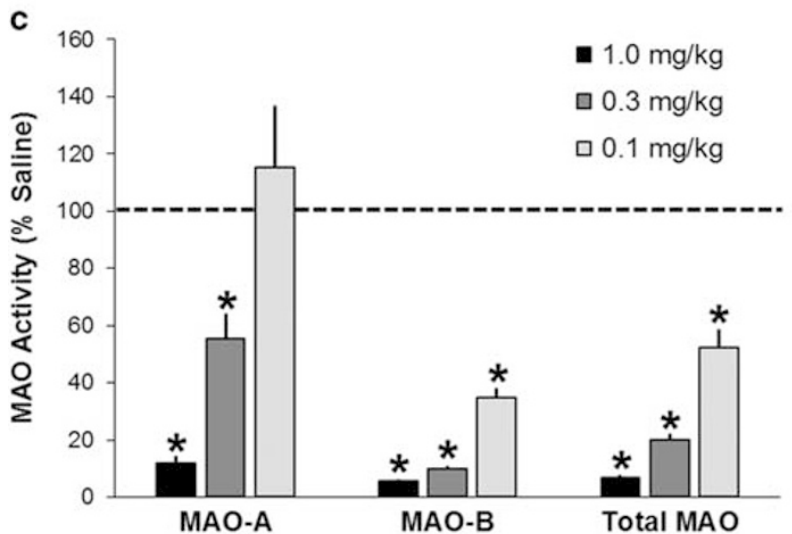

b
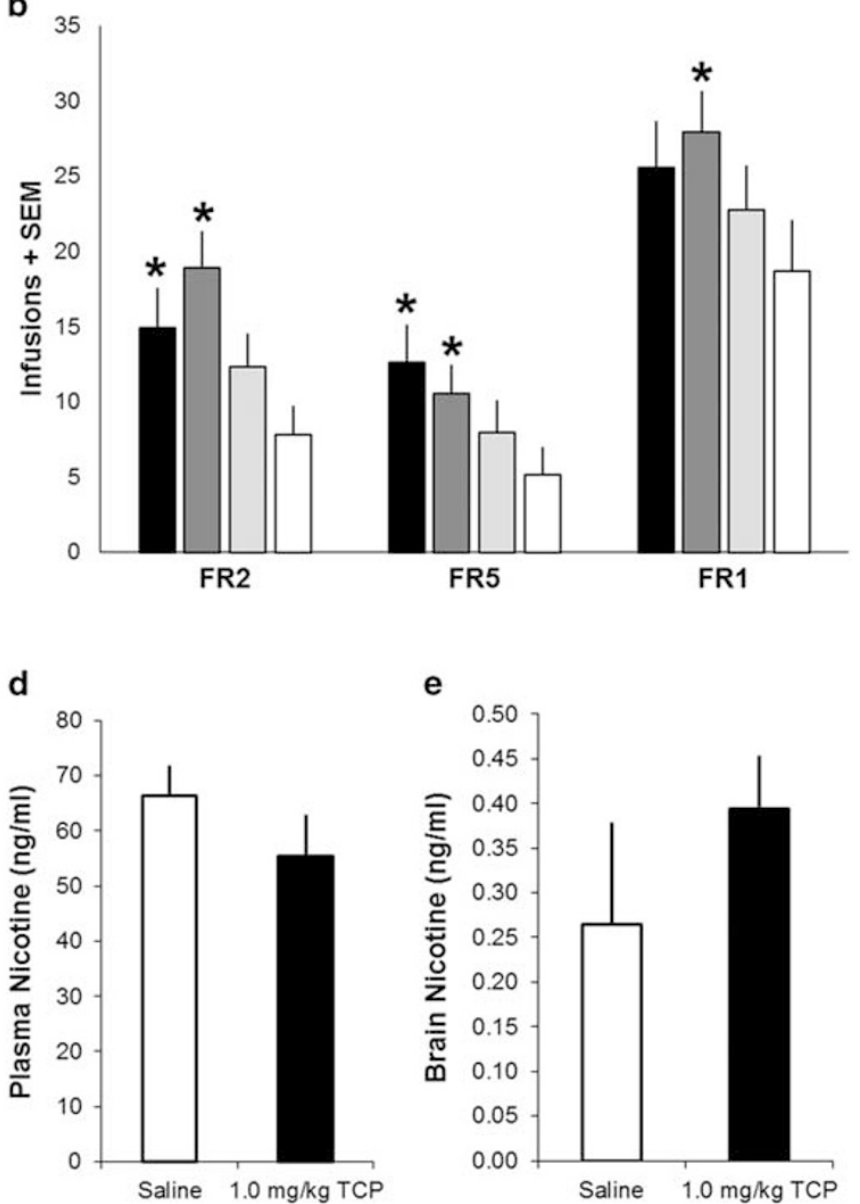

e

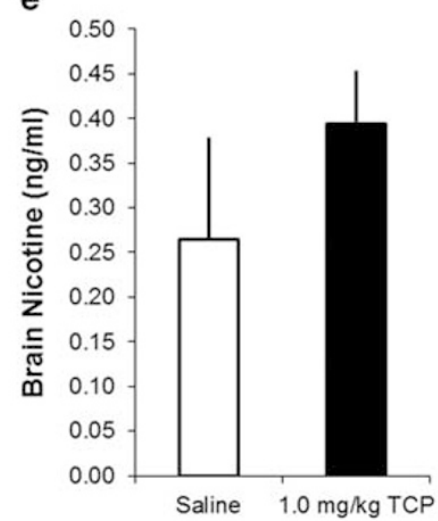

Figure 3 Impact of a range of TCP doses on nicotine self-administration on an FR2 schedule of reinforcement (a), on a range of FR schedules (b), and on MAO activity following the FRI portion of the experiment (c). The impact of $1.0 \mathrm{mg} / \mathrm{kg}$ TCP on nicotine levels in the blood and brain (22 infusions in I h, $n=6$ /group) is shown in (d) and (e). A significant difference from rats receiving pre-session injections of saline is represented by *

0.3-mg/kg TCP groups (1.0 mg/kg: $88 \% 0.3 \mathrm{mg} / \mathrm{kg}: 43 \%)$, but there was no significant change in MAO-A activity in the $0.1-\mathrm{mg} / \mathrm{kg}$ TCP group. There was no significant effect of $1.0 \mathrm{mg} / \mathrm{kg}$ TCP on plasma or brain nicotine levels in rats receiving non-contingent infusions of nicotine $(10 \mu \mathrm{g} / \mathrm{kg} /$ infusion, 22 infusions over $1 \mathrm{~h})$ ( $p s>0.05$; Figure $3 \mathrm{~d}$ and $\mathrm{e})$.

\section{Experiment 4: Impact of MAO Inhibition on Threshold for Reinforcement Enhancement}

A $2 \times 2 \times 3(\mathrm{TCP} /$ Saline $\times$ Nicotine/Saline $\times 3$ nicotine dose levels) ANOVA revealed a significant main effect of nicotine dose $(F(2,60)=47.80, p<0.001)$, a main effect of nicotine/ saline injection $(F(1,30)=5.83, p=0.022)$, and a nicotine/ saline injection $\times$ nicotine dose interaction $(F(2,60)=8.89$, $p=0.003)$, but no main effect of TCP, or interactions with TCP $(p s>0.05)$ (Figure 4). Follow-up independent-samples $t$-tests revealed that rats receiving both TCP and nicotine earned more VS presentations than rats receiving nicotine but not TCP at both the 0.03- $(t(16)=2.61, p=0.019)$ and $0.1-\mathrm{mg} / \mathrm{kg}$ nicotine doses $(t(16)=2.297, p=0.035)$. The pattern was similar for active responding, but there were no significant effects with TCP for inactive responding. These results suggest that $\mathrm{MAO}$ inhibition combined with a low dose of nicotine increased the reinforcing value of the VS more than low-dose nicotine without MAO inhibition.

\section{DISCUSSION}

The present experiments showed that (1) MAO inhibition increased sensitivity to the reinforcing effects of nicotine such that rats receiving injections of an MAO inhibitor earned more infusions at low nicotine doses and earned fewer infusions at high nicotine doses, (2) inhibition of MAO-A, but not MAO-B, increased self-administration of a low nicotine dose, (3) partial MAO inhibition increased responding for a low nicotine dose, and (4) MAO inhibition decreased the threshold for reinforcement enhancement. These findings replicate earlier work showing that MAO inhibition increases the reinforcing value of low nicotine doses and extend it by further characterizing the impact of $\mathrm{MAO}$ inhibition on the primary reinforcing and reinforcement enhancing effects of nicotine.

The results of Experiment 1 showed that MAO inhibition shifts the dose-response curve for nicotine to the left, such that it increases the reinforcing value of low nicotine doses and decreases the number of infusions earned at high 
nicotine doses. On a PR schedule, TCP increased the number of infusions earned at a low nicotine dose, but the number of infusions earned of a high nicotine dose remained unchanged. These data are consistent with multiple reports showing that TCP increases low-dose nicotine selfadministration as well as with data reported by Guillem et al (2005) showing that rats receiving $1.5 \mathrm{mg} / \mathrm{kg}$ TCP earned fewer infusions than rats receiving saline at a high nicotine dose $(80 \mu \mathrm{g} / \mathrm{kg} / \mathrm{infusion})$. The mechanism responsible for this leftward shift in the nicotine dose-response curve is unclear. Results presented in Experiment 3 showed that rats receiving non-contingent infusions of a low nicotine dose did not significantly differ on levels of nicotine in brain or blood, suggesting that differences in nicotine absorption and distribution are unlikely to be responsible for the shift in the nicotine dose-response curve. Furthermore, MAO inhibition does not simply increase sensitivity to all reward, as we show in Experiment 4 that TCP alone failed to increase the reinforcing value of a moderately-reinforcing VS. These data are consistent with previous data showing that MAO inhibition did not increase the reinforcing value of cocaine (Villegier et al, 2007) or food reward (Guillem et al, 2005).

Data from Experiment 2 confirm that it is inhibition of MAO-A, and not the inhibition of MAO-B, that increases self-administration of low nicotine doses. Guillem et al (2006) previously reported that clorgyline (a selective MAO-A inhibitor), and not selegiline (a selective MAO-B inhibitor), increased low-dose nicotine self-administration. They used doses of clorgyline and selegiline that were expected to be selective, but they did not confirm that by measuring MAO activity (Guillem et al, 2006). The present data extend their findings by showing that rats receiving clorgyline $(1.0 \mathrm{mg} / \mathrm{kg})$, but not rasagiline $(0.05)$, had increased low-dose nicotine self-administration compared with rats receiving pre-session injections of saline. MAO activity data from the same rats confirm that in rats receiving clorgyline, MAO-A activity was inhibited by $~ 91 \%$ with no impact on MAO-B. Conversely, in rats receiving rasagiline, MAO-B activity was inhibited by $\sim 94 \%$ and MAO-A activity was not inhibited, but was actually increased; this increase in MAO-A activity, which we have observed in other unpublished studies that included lower doses of rasagiline, complicates the interpretation of this experiment since the effect of MAO-B inhibition may be offset by an increase in MAO-A activity. The cause of this increase in MAO-A activity among rats receiving injections of rasagiline is unknown, but studies in cell culture suggest that rasagiline, and also another commonly used MAO-B inhibitor selegiline, increases the expression of MAO-A via a mechanism unrelated to MAO-B inhibition (Inaba-Hasegawa et al, 2013). Still, the current data suggest that cigarette smoke constituents that inhibit MAO-A, but not MAO-B, may increase reinforcement by low nicotine doses.

In chronic cigarette smokers, the MAO-A isozyme is suppressed by an average of $28 \%$, but is highly variable among smokers (Fowler et al, 1996a, b). Furthermore, the degree to which different types of tobacco and cigarette brands have been shown to inhibit MAO varies (Lewis et al, 2012). In Experiment 3, MAO-A was inhibited by $43 \%$ on average in the rats receiving $0.3 \mathrm{mg} / \mathrm{kg} \mathrm{TCP}$, and these rats earned significantly more nicotine infusions than rats receiving saline injections at all FRs. These data are consistent with previous data from our laboratory showing that MAO-A was inhibited by $44 \%$ at the time of selfadministration in rats receiving TCP injections $(1.0 \mathrm{mg} / \mathrm{kg})$ $23 \mathrm{~h}$ before the session and these rats earned significantly more infusions than rats not receiving TCP injections (Smith et al, 2015). MAO-A was not significantly inhibited in rats receiving $0.1 \mathrm{mg} / \mathrm{kg} \mathrm{TCP}$, and there was no significant effect of this dose at any FR. Together, these data suggest constituents that partially inhibit MAO-A, as is the case for chronic smokers, are likely to increase the reinforcing value of low nicotine doses. Also, variability in the degree of MAO-A inhibition may be related to variability in the reinforcing efficacy of low nicotine doses.

Experiment 4 is the first to investigate the impact of MAO inhibition on the reinforcement enhancing effects of nicotine. We used a procedure that is well established in our laboratory, in which rats respond for a moderately reinforcing VS and receive non-contingent nicotine (Caggiula et al, 2009; Donny et al, 2003; Rupprecht et al, 2015; Weaver et al, 2012). We showed that MAO inhibition decreased the threshold nicotine dose required for reinforcement enhancement. Rats receiving TCP $(1.0 \mathrm{mg} / \mathrm{kg})$ and low nicotine doses ( 0.03 and $0.1 \mathrm{mg} / \mathrm{kg}$ s.c.) earned more VS presentations than rats receiving nicotine but no TCP. These nicotine doses are typically subthreshold for reinforcement enhancement (unpublished laboratory observations). It is important to note that reinforcement enhancement may be partially or fully responsible for the effect of MAO inhibition on infusions earned in Experiments 1-3. In Experiments $1-3$, infusions were paired with an initially neutral stimulus (15-s cue light) (Palmatier et al, 2008), which over time likely becomes a conditioned reinforcer (Palmatier et al, 2007). After the stimulus is established as a reinforcer, low doses of nicotine in the presence of MAO inhibition may act to enhance the value of this reinforcer when these doses would be below the threshold for reinforcement enhancement without MAO inhibition. Consistent with this interpretation, differences in self-administration between rats receiving an MAO inhibitor and rats not receiving an MAO inhibitor consistently took 5-7 days to develop (Figures 2a and 3a), which would be the case if it was necessary for the cue to develop reinforcing value through its pairing with nicotine. It is unclear why the impact of MAO inhibition is delayed

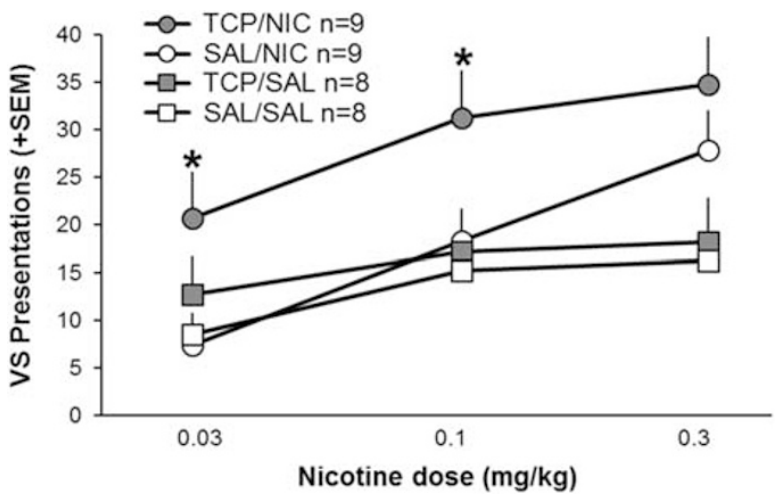

Figure 4 Average number of VS presentations earned on the last session at each nicotine dose. A significant difference between the TCP/NIC group and the SAL/NIC group is indicated by * 
given that inhibition of MAO likely occurs on the first day, but one possibility is that it takes time for the cue to become a conditioned reinforcer, which is then enhanced by nicotine. Other possible reasons for the delayed impact of MAO inhibition could be the time required to learn the behavioral response, or potentially a downstream effect of MAO inhibition.

These data are relevant as the FDA considers a potential policy regulating the nicotine content of cigarettes. MAO inhibition may have very little impact on current smokers, as there was little effect of MAO inhibition on nicotine selfadministration at doses traditionally considered to be the peak of the dose-response curve. However, if nicotine is reduced, then constituents that inhibit MAO-A are likely to increase the reinforcing value of nicotine and could shift the threshold for maintaining smoking behavior. Clinical trials using research cigarettes with very low nicotine contents likely contain MAO-inhibiting constituents that are similar to conventional cigarettes, meaning that nicotine reduction is already being evaluated in the presence of MAO inhibition. However, the level of MAO inhibition can vary across cigarette and tobacco types (Lewis et al, 2012), meaning that the FDA may still need to consider regulating the allowable MAO inhibition to ensure that the threshold is not shifted by cigarettes that have higher than normal levels of these constituents. Researchers should consider measuring MAOA activity in clinical trials on nicotine reduction.

\section{FUNDING AND DISCLOSURE}

Research reported in this publication was supported by the National Institute on Drug Abuse and FDA Center for Tobacco Products (CTP) (U54 DA031659 awarded to ECD). The funding source had no role other than financial support. The content is solely the responsibility of the authors and does not necessarily represent the official views of the NIH or the Food and Drug Administration. Funding for Tracy Smith was provided by the National Institute on Drug Abuse (F31 DA037643) and the National Cancer Institute (T32 CA186783). The authors declare no conflict of interest.

\section{ACKNOWLEDGMENTS}

Thanks to Joshua Alberts, Samuel Gutherz, Emily Pitzer, and Elizabeth Shupe for their extensive help in conducting experimental sessions. Thanks to undergraduates in the laboratory including E Corina Andriescu, Kayla Convry, Dora Danko, Mackenzie Meixner, Jessica Pelland, Hangil Seo, Nicole Silva, Isha Vasudeva, and Marisa Wallas.

\section{REFERENCES}

Arnold MM, Loughlin SE, Belluzzi JD, Leslie FM (2014). Reinforcing and neural activating effects of norharmane, a non-nicotine tobacco constituent, alone and in combination with nicotine. Neuropharmacology 85: 293-304.

Bardo MT, Green TA, Crooks PA, Dwoskin LP (1999). Nornicotine is self-administered intravenously by rats. Psychopharmacology (Berl) 146: 290-296.

Belluzzi JD, Wang R, Leslie FM (2005). Acetaldehyde enhances acquisition of nicotine self-administration in adolescent rats. Neuropsychopharmacology 30: 705-712.
Benowitz NL, Henningfield JE (1994). Establishing a nicotine threshold for addiction. The implications for tobacco regulation. N Engl J Med 331: 123-125.

Brennan KA, Crowther A, Putt F, Roper V, Waterhouse U, Truman P (2015). Tobacco particulate matter self-administration in rats: differential effects of tobacco type. Addict Biol 20: 227-235.

Buffalari DM, Marfo NY, Smith TT, Levin ME, Weaver MT, Thiels E et al (2014). Nicotine enhances the expression of a sucrose or cocaine conditioned place preference in adult male rats. Pharmacol Biochem Behav 124: 320-325.

Caggiula AR, Donny EC, Palmatier MI, Liu X, Chaudhri N, Sved AF (2009). The role of nicotine in smoking: a dual-reinforcement model. Nebr Symp Motiv 55: 91-109.

Clemens KJ, Caille S, Stinus L, Cador M (2009). The addition of five minor tobacco alkaloids increases nicotine-induced hyperactivity, sensitization and intravenous self-administration in rats. Int $J$ Neuropsychopharmacol 12: 1355-1366.

Costello MR, Reynaga DD, Mojica CY, Zaveri NT, Belluzzi JD, Leslie FM (2014). Comparison of the reinforcing properties of nicotine and cigarette smoke extract in rats. Neuropsychopharmacology 39: 1843-1851.

Depootere R, Li D, Lane J, Emmett-Oglesby M (1993). Parameters of self-administration of cocaine in rats under a progressive-ratio schedule. Pharmacol Biochem Behav 45: 539-548.

Donny EC, Chaudhri N, Caggiula AR, Evans-Martin FF, Booth S, Gharib MA et al (2003). Operant responding for a visual reinforcer in rats is enhanced by noncontingent nicotine: implications for nicotine self-administration and reinforcement. Psychopharmacology (Berl) 169: 68-76.

Donny EC, Denlinger RL, Tidey JW, Koopmeiners JS, Benowitz NL, Vandrey RG et al (2015). Randomized trial of reduced-nicotine standards for cigarettes. $N$ Engl J Med 373: 1340-1349.

Fowler JS, Volkow ND, Wang GJ, Pappas N, Logan J, MacGregor R et al (1996a). Inhibition of monoamine oxidase B in the brains of smokers. Nature 379: 733-736.

Fowler JS, Volkow ND, Wang GJ, Pappas N, Logan J, Shea C et al (1996b). Brain monoamine oxidase A inhibition in cigarette smokers. Proc Natl Acad Sci USA 93: 14065-14069.

Guillem K, Vouillac C, Azar MR, Parsons LH, Koob GF, Cador M et al (2005). Monoamine oxidase inhibition dramatically increases the motivation to self-administer nicotine in rats. J Neurosci 25: 8593-8600.

Guillem K, Vouillac C, Azar MR, Parsons LH, Koob GF, Cador M et al (2006). Monoamine oxidase A rather than monoamine oxidase $\mathrm{B}$ inhibition increases nicotine reinforcement in rats. Eur J Neurosci 24: 3532-3540.

Hatsukami DK, Perkins KA, Lesage MG, Ashley DL, Henningfield JE, Benowitz NL et al (2010). Nicotine reduction revisited: science and future directions. Tob Control 19: e1-10.

Inaba-Hasegawa K, Akao Y, Maruyama W, Naoi M (2013). Rasagiline and selegiline, inhibitors of type $\mathrm{B}$ monoamine oxidase, induce Type A monoamine oxidase in human $\mathrm{SH}-$ SY5Y cells. J Neural Transm 120: 435-444.

Lewis AJ, Truman P, Hosking MR, Miller JH (2012). Monoamine oxidase inhibitory activity in tobacco smoke varies with tobacco type. Tob Control 21: 39-43.

Lotfipour S, Arnold MM, Hogenkamp DJ, Gee KW, Belluzzi JD, Leslie FM (2011). The monoamine oxidase (MAO) inhibitor tranylcypromine enhances nicotine self-administration in rats through a mechanism independent of MAO inhibition. Neuropharmacology 61: 95-104.

Murphy SE, Park SS, Thompson EF, Wilkens LR, Patel Y, Stram DO et al (2014). Nicotine $\mathrm{N}$-glucuronidation relative to $\mathrm{N}$-oxidation and C-oxidation and UGT2B10 genotype in five ethnic/racial groups. Carcinogenesis 35: 2526-2533. 
Palmatier MI, Coddington SB, Liu X, Donny EC, Caggiula AR, Sved AF (2008). The motivation to obtain nicotine-conditioned reinforcers depends on nicotine dose. Neuropharmacology 55: 1425-1430.

Palmatier MI, Evans-Martin FF, Hoffman A, Caggiula AR, Chaudhri N, Donny EC et al (2006). Dissociating the primary reinforcing and reinforcement-enhancing effects of nicotine using a rat self-administration paradigm with concurrently available drug and environmental reinforcers. Psychopharmacology (Berl) 184: 391-400.

Palmatier MI, Liu X, Matteson GL, Donny EC, Caggiula AR, Sved AF (2007). Conditioned reinforcement in rats established with self-administered nicotine and enhanced by noncontingent nicotine. Psychopharmacology (Berl) 195: 235-243.

Rodgman A, Perfetti TA (2013). The Chemical Components of Tobacco and Tobacco Smoke, 2nd edn. CRC Press, Taylor \& Francis Group: Boca Raton, FL, USA.

Rupprecht LE, Smith TT, Schassburger RL, Buffalari DM, Sved AF, Donny EC (2015). Behavioral mechanisms underlying nicotine reinforcement. Curr Top Behav Neurosci 24: 19-53.

Smith TT, Levin ME, Schassburger RL, Buffalari DM, Sved AF, Donny EC (2013). Gradual and immediate nicotine reduction result in similar low-dose nicotine self-administration. Nicotine Tob Res 15: 1918-1925.

Smith TT, Schaff MB, Rupprecht LE, Schassburger RL, Buffalari DM, Murphy SE et al (2015). Effects of MAO inhibition and a combination of minor alkaloids, beta-carbolines, and acetaldehyde on nicotine self-administration in adult male rats. Drug Alcohol Depend 155: 243-252.

U.S. Congress (2009). Family Smoking Prevention and Tobacco Control Act (H.R. 1256). Available at: https://www.govtrack.us/ congress/bills/111/hr1256/text. Accessed 23 February 2016.

Villegier AS, Belluzzi JD, Leslie FM (2011). Serotonergic mechanism underlying tranylcypromine enhancement of nicotine selfadministration. Synapse 65: 479-489.

Villegier AS, Blanc G, Glowinski J, Tassin JP (2003). Transient behavioral sensitization to nicotine becomes long-lasting with monoamine oxidases inhibitors. Pharmacol Biochem Behav 76: 267-274.

Villegier AS, Lotfipour S, McQuown SC, Belluzzi JD, Leslie FM (2007). Tranylcypromine enhancement of nicotine self-administration. Neuropharmacology 52: 1415-1425.

Villegier AS, Salomon L, Granon S, Changeux JP, Belluzzi JD, Leslie FM et al (2006). Monoamine oxidase inhibitors allow locomotor and rewarding responses to nicotine. Neuropsychopharmacology 31: 1704-1713.

Weaver MT, Geier CF, Levin ME, Caggiula AR, Sved AF, Donny EC (2012). Adolescent exposure to nicotine results in reinforcement enhancement but does not affect adult responding in rats. Drug Alcohol Depend 125: 307-312.

Wing VC, Shoaib M (2010). A second-order schedule of food reinforcement in rats to examine the role of $\mathrm{CB} 1$ receptors in the reinforcement-enhancing effects of nicotine. Addict Biol 15: 380-392. 